\title{
Implementation of Privacy Preservation Scheme for Data Center Networks
}

\author{
Madhuri P. Gole, Prof. Sachin Vyawahare
}

\begin{abstract}
Advances in information escalated figuring and elite registering encourage fast scaling of data server farm systems, bringing about a developing assortment of research investigating new system designs that improve adaptability, cost viability and execution. to understanding the exchange of distinctive system structures couldn't just assist information with focusing administrator's improvement of arrangements, yet in addition help framework developer to streamline applications running over them. In this paper presenting the multiple routing configuration protocol and break down its exhibition as for adaptability, reinforcement way lengths, and burden appropriation after a disappointment. We additionally show how a gauge of the traffic requests in the system can be utilized to improve the dispersion of the recouped traffic, and subsequently diminish the odds of clog when multiple routing configuration is utilized.
\end{abstract}

Index Terms - Data Center Networks, Multiple Routing configurations, Network.

\section{INTRODUCTION}

Now a day the data centers plays an important role to providing the various services to store the large amount of data for the business intelligence such as content mining, text mining etc. To design the effective and efficient data centers in distributed environment. Data center is the way toward setting up and the whole system and various networking devices are connected to each other to provide the various services to the users.

It empowers an advanced association of data centers to providing the communication between the data centers and network nodes over the internet.

The data center gives numerous techniques for processing, storage, and resource utilization but the existing has some limitation. So as to accomplish this objective, data center should ensure every one of the assets in work in show is

Manuscript revised on November 02, 2019 and published on November 20, 2019

Madhuri P. Gole, Master of Engineering in Computer Science and Information Technology from Sanmati Engineering College, Washim.

Prof. Sachin Vyawahare Asst. Professor and HOD of Computer

Department at Sanmati Engineering College Washim (MH) accomplished the plan and arrangement. Especially, distributed network is distinguished to integrate with the mix of registering and information frameworks to give an adaptable, versatile and economical beneficial way to deal with help the consistently developing basic Information Technology requirement of the business intelligence and the overall population.

Now for development of distributed environment requires to increases the data centers, in this way to design the strong network to connect the different servers in such way that the network become very cost effective and easy to troubleshoot.

So now present data centers has much limitation and facing the various problems. Number of scholars have been developed the techniques to solve the issue related to the data centers such as to design the various protocols, resource optimizing etc.

In light of their past work and present research, aim of this paper is to examine the data centers and also considered the background details related to the data centers. In addition, in view of cautious perceptions we likewise advance some contemplations and recommendations on the current problems of data servers. The essential objective of this paper presenting the current arranges difficulties and practical arrangements that are related with server farm organizing.

\section{LITERATURE REVIEW}

\section{A. Review Stage}

Ting Wang et. al. [1] examine the data center server and gives the outline and investigation of the writing covering different research regions such as server farm arrange interconnection structures, organize conventions for server farm systems, and system asset partaking in cloud server. Study with review on server farm systems and together with its prerequisites explore the server farm arrange structures.

Alan Shieh et. al. [2] studied the Seawall, a system data transmission portion plot that partitions organize limit dependent on a user indicated arrangement. Proposed system figures and authorizes assignments by burrowing traffic by clog controlled, to point the multiple direct burrows. The subsequent distributions stay stable paying little respect to the quantity of streams, conventions, or goals in the application's traffic blend. Not at all like interchange recommendations, Seawall effectively underpins dynamic approach and scales up the quantity of uses and stir of the present server farms. Through assessment of a model, we 
show that Seawall includes minimal overhead and accomplishes solid execution confinement.

Pries R et. al. [3] presents various data server models and look at them with respect to their capacity utilization. The outcomes show that there are a few contrasts which ought not be disregarded and that with just minor changes of the design, it is conceivable to spare a gigantic measure of power.

Fan Yao et. al. [4] studied the similar investigation of a few surely understood data server farm organize structures utilizing significant measurements, and outcomes on various topology. they shows exchange offs between the topology and present ramifications on reasonable data server usage.

Theophilus Benson et. al. [5] analyze the scope of uses conveyed in the data server farms and the stream level and packet of transmission assets of these applications, and their effect on arrange use, connect use, blockage, and bundle drops. We depict the ramifications of the watched traffic designs for server farm inward traffic building just as far as of late proposed structures for server farm systems.

Justin Meza et. al. [6] introduced huge amount of longitudinal investigation of data server farm organize dependability dependent on information gathered from the creation of network framework of Facebook. The examination traverses a great many intra server farm organize episodes crosswise over seven years, and eighteen months of bury server farm arrange occurrences. To show how the unwavering quality attributes of various system plans and distinctive system gadget types show as system episodes and influence the product frameworks that utilization the system.

\section{Proposed System}

The proposed system ensures the recovery of all networks down situations, utilizing a solitary system to deal with both connection and sensor node fail and without realizing the underlying reason of network fail. Multiple Routing Configurations is carefully connectionless protocol. Multiple Routing Configuration depends on extra steering data in the switches, and permits packet for sending to proceed on an elective yield connect following the discovery of network fail. The proposed system can be categorized of three different sections

[1] Client section

[2] Routers section

[3] Server section

\section{Client section}

- In this section transfer the information to server through routers.

- It will give easy to use interface to transfer the information to the necessary node.

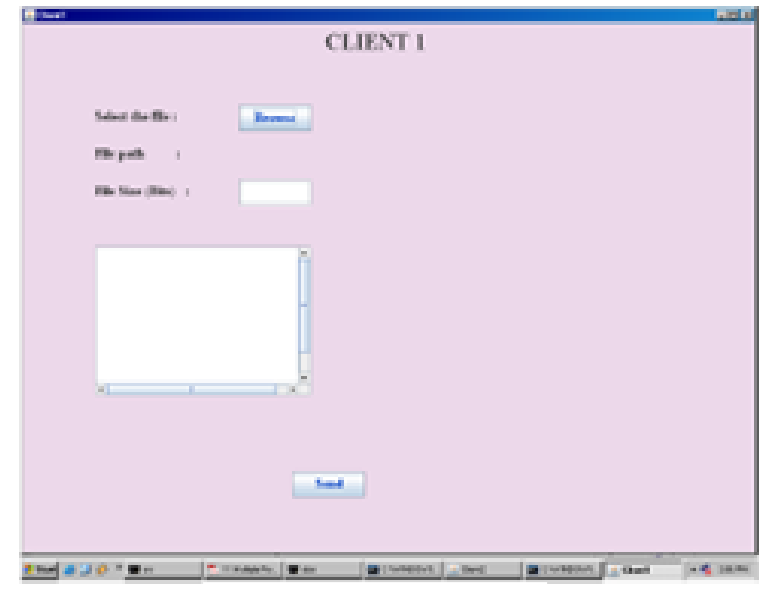

Figure 1: Client 1

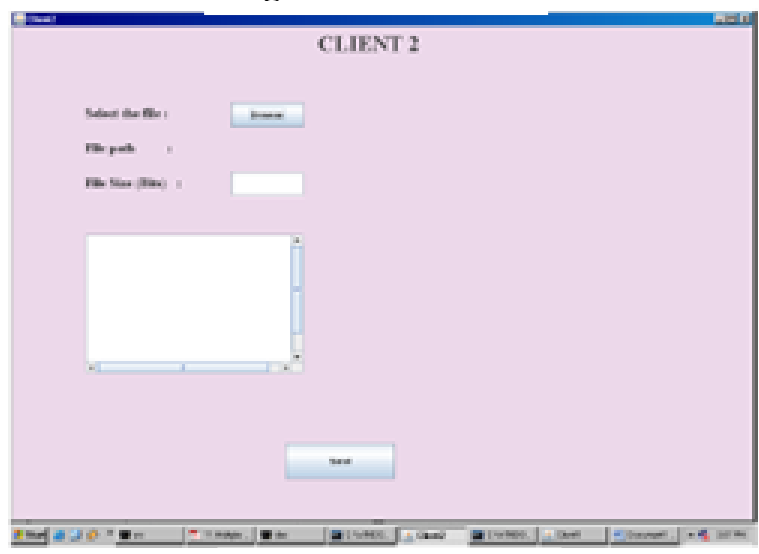

Figure 2: Client 2

\section{Router section}

- This are set in the middle of server and customer to move the information.

- Whenever customer transfers the information to the server it will go through any one switch.

- If the switch is bombed the information will be moved through another switch to decrease the framework failure situation

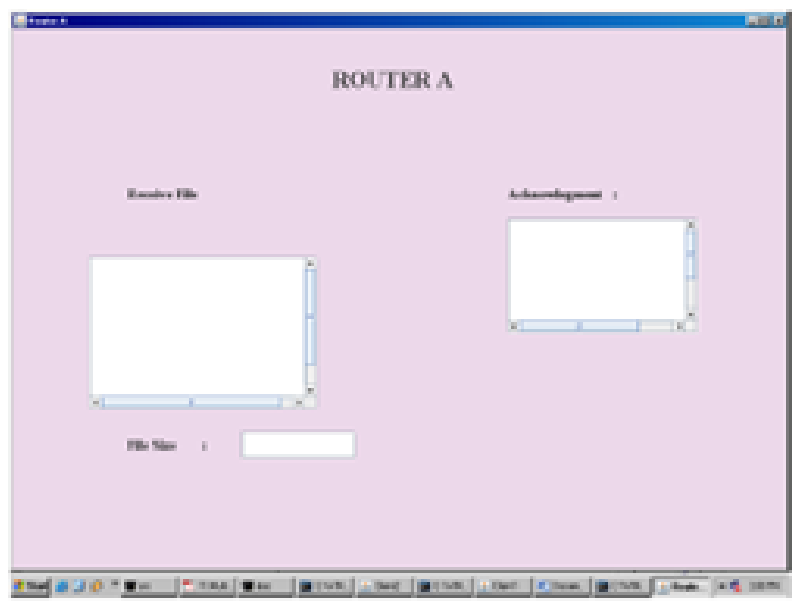

Figure 3: Router A 


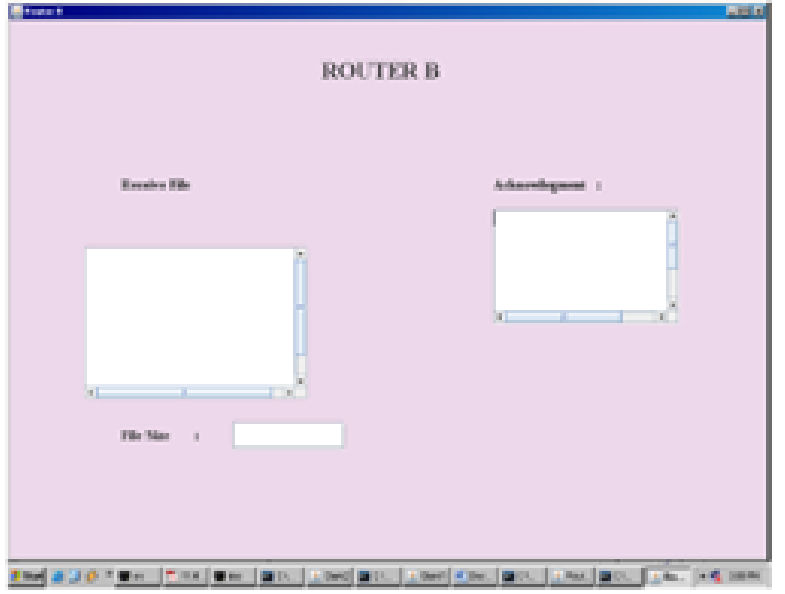

Figure 4: Router B

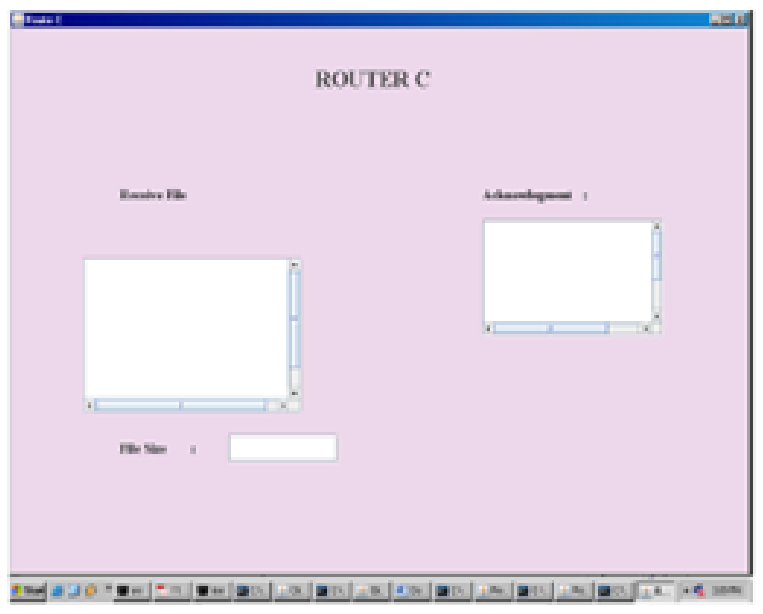

Figure 5: Router C

\section{Server Module}

It will get the information send by the customer which originated from the dynamic switch.

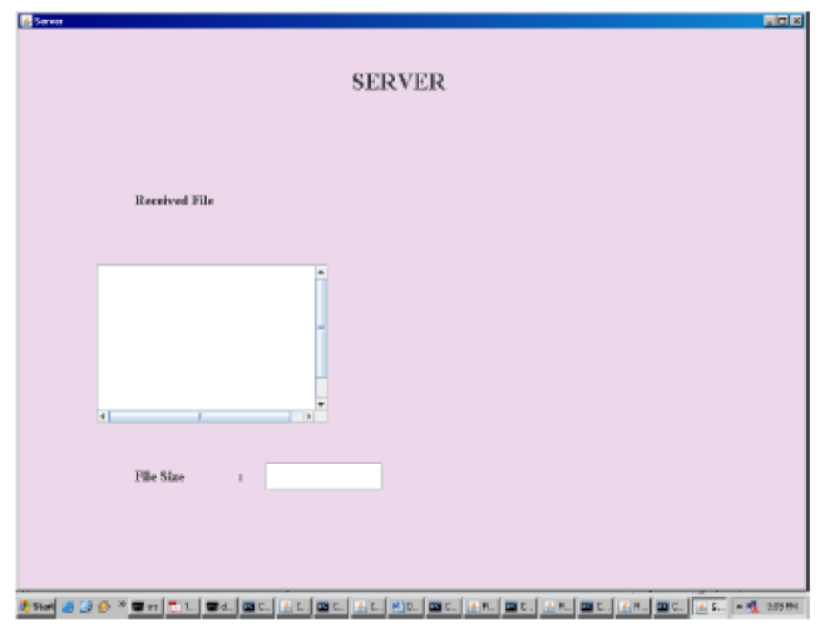

Figure 6: Server

\section{Conclusion}

Data Server center are playing an increasingly more significant job in the new time of could processing, unavoidably went with an ever-increasing number of difficulties and issues in different viewpoints. In this paper, we gave an extensive survey on the related research writing on server farms. We talked about numerous novel proposed plans and procedures in various research territories extending from data center topologies, transport conventions, to arrange sharing instruments in server farms.

\section{REFERENCES}

[1] Ting Wang, et. aal. (2014)"Rethinking the Data Center Networking: Architecture, Network Protocols, and Resource Sharing, IEEE Access, Volume 2, 2014.

[2] Alan Shieh, Srikanth Kandula, Albert GreenbergV, Changhoon KimV, Bikas Saha, "Sharing the Data Center Network".

[3] Pries R et. al. . (2012) Power Consumption Analysis of Data Center Architectures. In: Rodrigues J.J.P.C., Zhou L., Chen M., Kailas A. (eds) Green Communications and Networking. GreeNets 2011. Lecture Notes of the Institute for Computer Sciences, Social Informatics and Telecommunications Engineering, vol 51. Springer, Berlin, Heidelberg.

[4] Fan Yao, Jingxin Wu, Guru Venkataramani, Suresh Subramaniam, "A Comparative Analysis of Data Center Network Architectures".

[5] Theophilus Benson, et. al. (2010) "Network Traffic Characteristics of Data Centers in the Wild", IMC'10, November 1-3, 2010, Melbourne, Australia.

[6] Justin Meza,et. al. (2018), "A Large Scale Study of Data Center Network Reliability", IMC '18, October 31-November 2, 2018, Boston, MA, USA, https://doi.org/10.1145/3278532.3278566

[7] M. Al-Fares, et. al. (2008), "A scalable, commodity data center network architecture," ACM SIGCOMM Comput. Commun. Rev., vol. 38, no. 4, pp. 63-74, [Online]. Available: http://doi.acm. org/10.1145/1402946.1402967

[8] G. D. Stamoulis et. al. (1991), "The efficiency of greedy routing in hypercubes and butterflies," in Proc. 3rd Annu. ACM Symp. Parallel Algorithms Archit. (SPAA), New York, NY, USA, pp. 248-259. [Online]. Available: http://doi.acm.org/10.1145/113379.113402

[9] Srikanth Kandula (2009), "The Nature of Datacenter Traffic: Measurements \& Analysis", IMC'09, November 4-6, 2009, Chicago, Illinois, USA.

[10] S. B. Akers et. al. (1989), "A group-theoretic model for symmetric interconnection networks," IEEE Trans. Comput., vol. 38, no. 4, pp. 555-566, Apr. 1989.

\section{AUTHORS PROFILE}

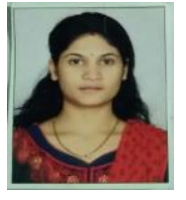

Miss Madhuri P. Gole completed Bachelor of Engineering in Computer Science and Engineering from Rajashri Shahu College of Engineering Buldhana, and pursuing Master of Engineering in Computer Science and Information Technology from Sanmati Engineering College, Washim.

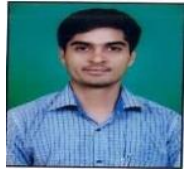

Prof. Sachin Vyawhare is working as Asst. Professor and HOD of Computer Department at Sanmati Engineering College Washim $(\mathrm{MH})$. He received $\mathrm{BE}$ degree and ME degree from S.G.B.A.U. Amaravati. His research interest includes networking, Operating System and Image Processing. 